\title{
Identification and modeling of the three rotational movements of a miniature coaxial helicopter
}

\author{
Bogdan Muresan', Silviu Folea', Ioan Nascu', Clara lonescu ${ }^{2}$ and Robin De Keyser ${ }^{2}$
}

\begin{abstract}
This paper presents the identification of a miniature coaxial helicopter system. First, the helicopter flying principles are described and the hardware setup of the developed platform is presented. Further, linear models are developed for the movements of the helicopter using prediction error identification methods. The results in this case are accurate and can be used for performant controller design in some operating points. But in order to model the complete dynamics of the helicopter, nonlinear models are developed using recurrent dynamic neural networks. In this case the models obtained present a higher accuracy compared with the linear case and also with the results published until now. In the end, the advantages of nonlinear modeling based on neural networks is emphasized and some conclusions are drawn.
\end{abstract}

Keywords

System identification, modeling, neural networks, coaxial helicopter

\section{Introduction}

Unmanned aerial vehicles (UAVs) have gained interest from the academic community worldwide in recent years, due to their numerous applications in civil and military applications, especially in missions which are not only impossible or dangerous for humans to accomplish but even for other types of devices. ${ }^{1-5}$

The flying devices ranging from $15 \mathrm{~cm}$ to $1 \mathrm{~m}$ in length with speeds between 5 and $20 \mathrm{~m} / \mathrm{s}$ are called micro (or miniature) aerial vehicles (MAV) and represent a special class of UAV. ${ }^{6-8}$ There are various configurations of aerial platforms, e.g. fixed-wing, quadrotors, helicopters, coaxial helicopters, and flying blimps, but the coaxial helicopters proved to be among the best suited for autonomous indoor flight. $^{9-11}$ The main reason for this is their ability for unique flight capabilities such as vertical takeoff/landing, hovering, slaloming, and pirouetting, properties that place them as the most complex among the UAVs. Their nature is multivariable by definition, with strong couplings between the variables and high nonlinearity. ${ }^{12,13}$

The autonomous miniature helicopters are especially adequate for dangerous and demanding activities such as high accuracy terrain mapping; traffic, volcano, or archeological site surveillance; power line monitoring; or target localization. In order to complete such missions, the vehicle should be able to maintain its stability while following a certain trajectory under the guidance of an embedded control algorithm. In consequence, the controller design is one of the major and significant steps that has to be completed in order to produce an autonomous and stable flying vehicle. Furthermore, a performant controller needs very accurate models of the system. Actually, recently, more and more evolved control algorithms have been used for controlling the UAVs: high accuracy in the models used for control design has become indispensable.

Several methods have been applied up to now for model-based control of UAVs, making system dynamics modeling a main issue. The first principle approach refers to physical modeling and involves the equation of motion using fundamental laws of mechanics and aerodynamics. For such a complex system as a coaxial helicopter, the resulting equations are high-order nonlinear coupled differential equations. This approach needs substantial knowledge and experience of both theory and design characteristics of all components of the flying device. For this

\footnotetext{
'Technical University of Cluj-Napoca, Romania

${ }^{2}$ Ghent University, Belgium

\section{Corresponding author:}

Bogdan Muresan, Department of Automation, Strada Constantin

Daicoviciu nr. 15, Cluj-Napoca 400020, Romania.

Email: bogdan.muresan@aut.utcluj.ro
} 
reasons it is difficult to accomplish, implying high cost in both time and money. ${ }^{14}$

But even in the work by Schafroth et al., ${ }^{14,15}$ linear models are proposed for control design because in most flying qualities studies, simple linear models are sufficient. ${ }^{16}$ Usually in hovering mode, a linear model is able to capture the essential dynamics of a helicopter. ${ }^{17}$ The drawback in this case is that multiple linear models and multiple controllers are required in order to cover all flight operating points.

The flight tests realised were made using a commercially available coaxial helicopter and an inertial measurement unit (IMU). ${ }^{18}$ The final purpose of the research that generated this work is to achieve a low-cost, autonomous, and stable flying device suitable for surveillance in narrow indoor spaces. A first part of the research project is presented here, i.e. the identification and modeling of a UAV system.

Both linear and nonlinear identification and modeling techniques are aproached. While the linear models were obtained using a prediction error method (PEM), recurrent dynamic neural networks were trained to approximate the behaviour of the coaxial helicopter in hovering. Previous works have proven that neural networks in modeling provide similar or better results to those obtained using first principal modeling; ${ }^{8,19}$ furthermore, neural networks are faster and do not require large computing power or complicated calculations. $^{20}$

The rest of the paper is organized as follows. In the second section the coaxial helicopter is presented in detail together with the equipment used for flight data acquisition and data processing. Section 3 presents a PEM, used for the identification of linear models of the three angular movements of the flying device. Further, in the next section, nonlinear recurrent dynamic neural networks are trained to predict the behaviour of the system. In Section 5 , all the results of the identification process are presented and the performances of the linear models are compared with those of the nonlinear models. Finally, some conclusions are drawn in Section 6.

\section{The UAV system}

The most common helicopter configuration is that with only one main rotor and a tail rotor. For this project a helicopter with a coaxial configuration was chosen due to its advantages over the classical configuration: it is more stable and more easy to maneuver during flight; it is also more compact since the tail rotor is not needed and it can carry a bigger payload using the same motor power. After choosing the configuration, in order to identify, model, and control a helicopter, one can choose to design and build a new one or to use one existing on the market. The solution chosen here was to acquire a helicopter from the market and to add extra devices necessary for the identification and control design. The entire UAV platform developed during this project is presented in Figure 1.

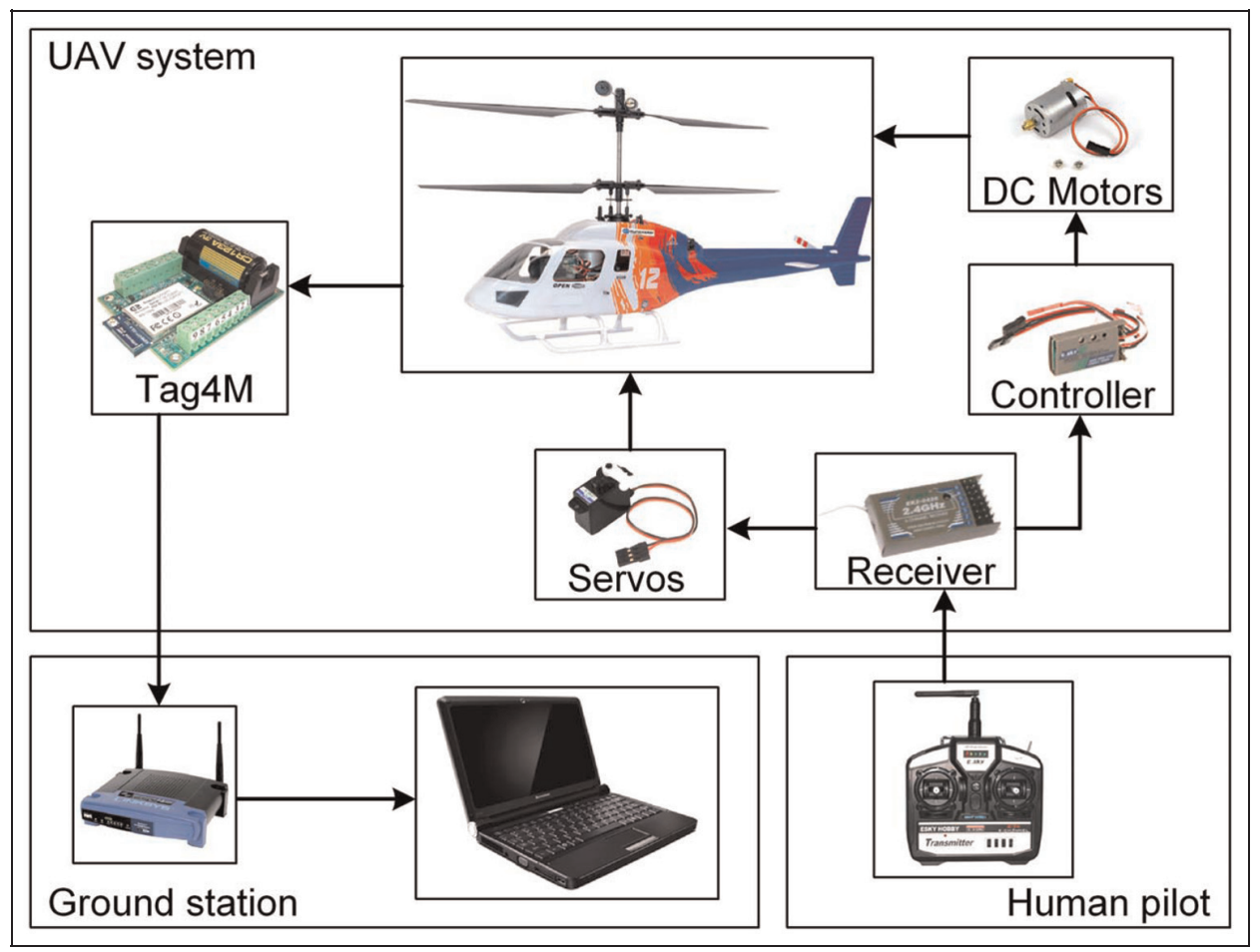

Figure I. UAV platform diagram. 


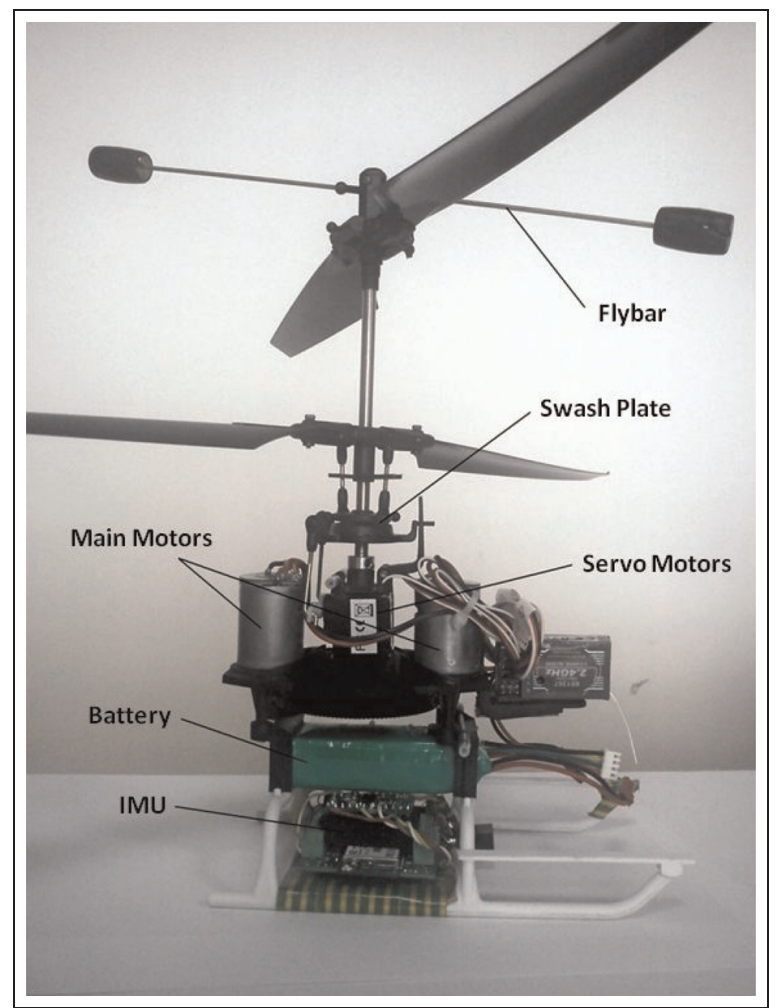

Figure 2. Big Lama coaxial miniature helicopter.

Coaxial helicopters use two sets of rotors that turn in opposite directions generating a pair of equal and opposite torques, where it will greatly reduce the gyroscopic effects which would otherwise impede obtaining an equilibrium position. This happens only when both sets of blades rotate with the same speed, which means the torques developed are equal, and, due to their opposite direction, the torques will cancel each other. This makes it possible to control the yaw by modifying the difference of the speeds of the two rotors and also the altitude by modifying the sum of two speeds.

\section{I. The Big Lama helicopter}

The helicopter chosen for this project was a Big Lama helicopter, manufactured by E-sky (Figure 2). It is one of the biggest helicopters found on the market, it is easy to fly, and it is able to carry the sensors and other hardware needed. The main characteristics are presented in Table 1.

The main element, that makes it easy to control the helicopter, is the swashplate, and this can be seen in Figure 2. This component permits the blades to tilt, and, as a result, the direction of the helicopter will change. The upper part of the swashplate rotates in the same time as the lower rotor, while the lower part is connected to the servomotors, which will change the tilt angle. This is how,
Table I. Big Lama physical specifications.

\begin{tabular}{ll}
\hline Main rotor diameter & $460 \mathrm{~mm}$ \\
Weight & $410 \mathrm{~g}$ \\
Length & $510 \mathrm{~mm}$ \\
Width & $110 \mathrm{~mm}$ \\
Height & $260 \mathrm{~mm}$ \\
Motors & Model $370(2$ installed $)$ \\
RC transmitter & 4 channels \\
Receiver & $2.4 \mathrm{GHz}$ \\
Servomotors & Digital $(2$ installed $)$ \\
Battery & II.IV $800 \mathrm{mAh} \mathrm{Li-polymer}$ \\
\hline
\end{tabular}

acting with the servomotor, an $x$-axis rotation is obtained (roll rotation), while acting with the second servomor a $y$ axis rotation is obtained (pitch rotation). Another component that directly influences the aerodynamics of the helicopter is the flybar (stabilization bar), which is meant to keep the helicopter in a stable position by acting as a damper for sudden changes in the rotation speed of the rotor. Both servomotors and also the two brushless DC motors that drive the rotors are powered by a lithium polymer battery. Supplementary, an IMU is needed in order to obtain the position and orientation of the helicopter. All the helicopter components are presented in Figure 2.

For full control the helicopter has to be steered around four axes: horizontal ( $x$ - and $y$-axis), in altitude (z-axis) and in heading $(\psi)$. There are four variables that can be controlled directly with the throttle, rudder, aileron, and elevator (see Figure 3). Because a sensor to measure the distance to the ground is not available at this moment, the identification of altitude (linear velocity on $z$-axis) was not possible yet.

Under the canopy of this model there is a receiver with six channels and an embedded control system. Only four channels are used, corresponding to the four movements of the helicopter mentioned above. For controlling pitch and roll movements two signals are received (elevator for pitch and aileron for roll). These control signals are transmitted directly to the servomotors, while the other control signals (rudder for yaw and throttle for the altitude) are transmitted first to the controller, where they are mixed with an internal gyroscop signal. Further, the controller will send pulse width modulation (PWM) signals to the two motors. The gain and the offset can be set at this controller, but the available information about these parameters is very poor.

It can be noticed that a decoupling is made, at the hardware level, on this type of coaxial helicopter. Normally, yaw and altitude movements are strongly coupled in a coaxial configuration, because both are driven at the same time by both DC motors. In our case, yaw is controlled directly with rudder and the altitude directly by throttle (see Figure 3). This means that the rudder represents the difference of the speeds of the two motors, while the 


\begin{tabular}{|c|c|c|}
\hline Elevator & \multirow{4}{*}{ Helicopter } & Pitch \\
\hline Aileron & & Roll \\
\hline Rudder & & Yaw \\
\hline Throttle & & Altitude \\
\hline
\end{tabular}

Figure 3. Coaxial helicopter's system inputs and outputs.

throttle is the sum of the two speeds. So, the decoupling of the two movements makes it possible to consider the helicopter as a system with four SISO subsystems (Figure 3). This is especially valid in hovering mode.

\subsection{Tag4M module and sensors}

For data acquisition during flight experiments a Tag4M module was used. The Tag4M device is a Wi-Fi data acquisition system, developed for low power consumption and mobile applications. By attaching sensors to its inputoutput terminal blocks in a similar manner as for a wired data acquisition device, the user can build a wireless proofof-concept sensor solutions for a wide range of applications. The system has the advantage of reduced dimensions $(4.7 \mathrm{~cm} \mathrm{x} 7.0 \mathrm{~cm})$ and of a limited weight of $50 \mathrm{~g}$, and can run on battery power, making it a portable solution. The dimensions of the system are reducible to $2.4 \times 4.0 \mathrm{~cm}$ and $15 \mathrm{~g}$; this is an important feature because the helicopter has a limited lifting power. It is a complete Wi-Fi networking solution, incorporating a 32-bit CPU, a memory unit, an eCos real-time operating system and a UDP or TCP/IP stack. Other included components are the analogic sensor interface, the power management unit, the hardware cryptographic accelerator, and the real-time clock.

The hardware architecture of the device is presented in Figure 4. At this version, five analog inputs, four digital output channels, and two serial ports are available. A custom interface was implemented to allow the acquisitions from two sources: 3-axis accelerations and 2-axis gyroscopes. ${ }^{18}$

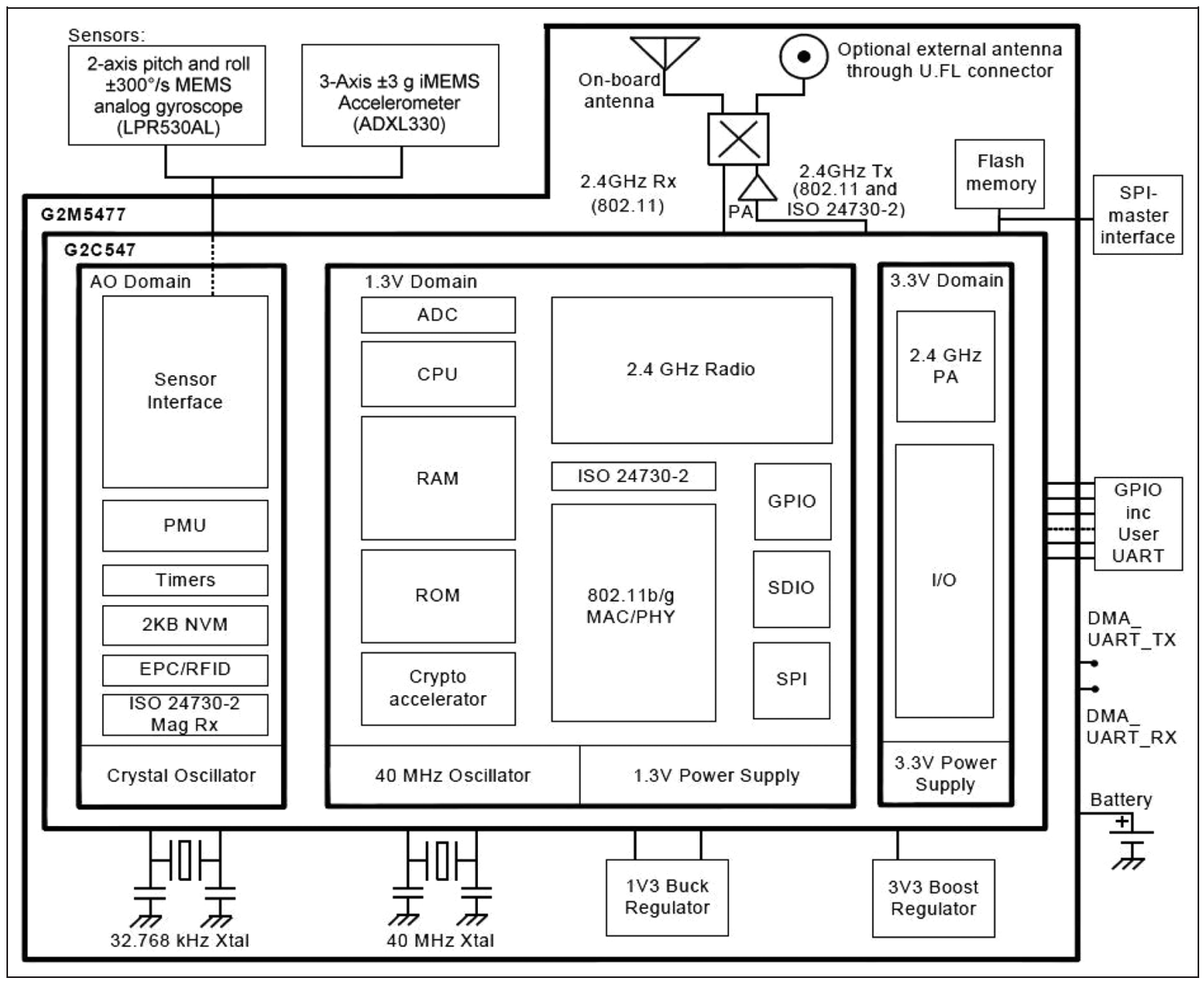

Figure 4. Hardware architecture of the Tag4M data acquisition system. 
The Tag4M device is running in power cycles, each cycle containing a period of data acquisition, a receiving period and a transmission time. The tag doesn't have a memory buffer, the data acquired being transmitted after each reading. An important feature is that the power consumption can be drastically reduced by combining the acquisition, processing, and transmission periods with sleeping periods.

By attaching a 2-axis gyroscope and 3-axis accelerometer sensors to the Tag4M board, it's possible to determine the helicopter's dynamic behaviour based on the data received. For this reason a LabVIEW application was developed and implemented on the ground station in order to acquire and scale the sensors data. The 3-axis accelerometer (ADXL330, iMEMS type from Analog Devices) allows the measurement of static or dynamic acceleration in the range $\pm 3 \mathrm{~g}$ on all three axes of interest. A supplementary low-pass analog filter was implemented using internal resistors from the acceleration sensor and capacitors with a bandwidth of $10 \mathrm{~Hz}$. The gyroscope chosen to be used integrates one actuator and one accelerometer in a single micro machined structure. LPR530AL is based on the Coriolis principle, and it is able to react when an angular rate is applied to the sensing element which is kept in continuous oscillating movement. It has a full scale of $\pm 300 \mathrm{deg} / \mathrm{s}$. Again for this sensor a low pass filter, with a cutoff frequency of $10 \mathrm{~Hz}$, was added on the board. The additional analog filtering is highly recommended for such an application because the main source of noise (the vibrations of the helicopter during flight) affecting the sensor data is of a higher frecquency than $20 \mathrm{~Hz}$ and it may cause the appearance of the aliasing effect.

One initialization step when the application starts is necessary for reading the data from the tag. During this stage, the tag sends a package containing the internet protocol (IP) received through the dynamic host configuration protocol (DHCP) from the access point (AP). This IP is used in the application for sending commands to the Tag4M device after the initialization step. The data are read in a loop and are validated if they are received from a previously known media access control (MAC) address which is used as a validation mask. The latency determined after performing a number of experiments lies between 5 and $20 \mathrm{~ms}$ and it depends on the RSSI signals values. The values of the latency are greater in case of a poor signal.

The utility of this device is proved by its characteristics: small dimensions, ultra-low power consumption, Wi-Fi transmission capabilities, and the number of input-output channels, both analog and digital. Moreover the 32-bit CPU permits the implementation of a controller while the four digital outputs allow direct control of the two DC motors and the two servomotors using PWM signals.

The Tag4M was mounted on the helicopter and during the flight experiments the sensor data was sent to the

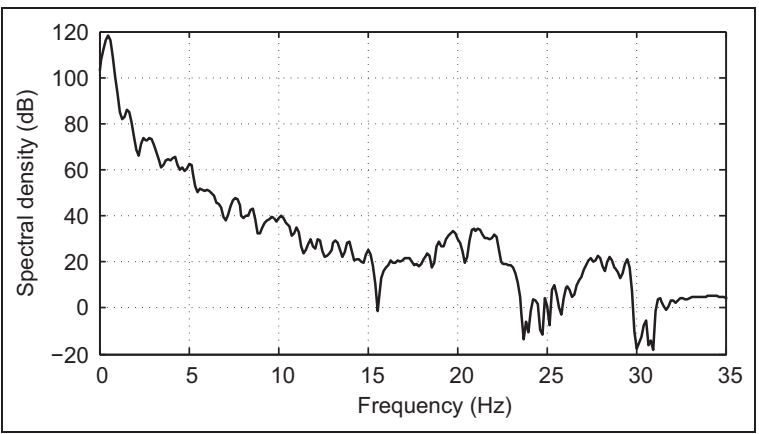

Figure 5. Spectral analysis of a gyroscope signal.

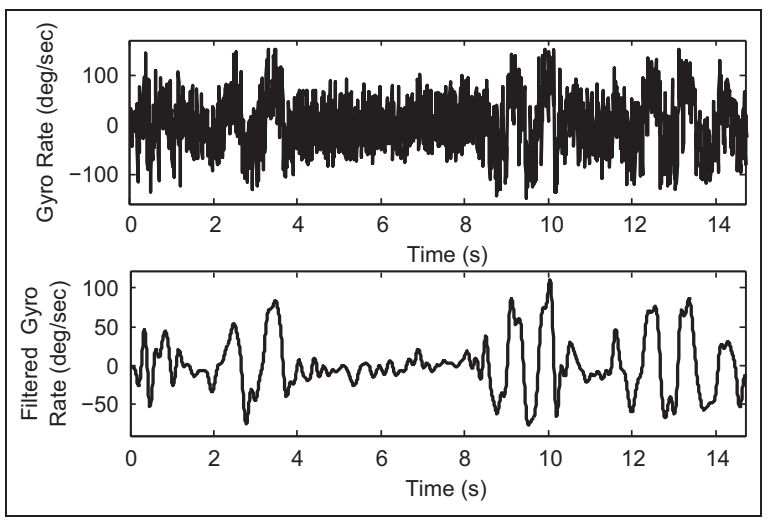

Figure 6. Result of digital filtering.

ground station via an access point (Figure 1). Through the LabVIEW application the flight data was saved in different files and afterwards was processed in Matlab.

A digital filter was used for supplementary software filtering of the sensor signals. Since the noise affecting the signals, caused especially by the vibrations, has a high frequency, a lowpass filter is needed. The filter chosen is a Butterworth of 2 nd order with a cutoff frequency of $5 \mathrm{~Hz}$ and the filtering of the data was made in both the forward and reverse directions in order to avoid a phase shift in the useful signal. The spectral analysis of a gyroscope signal is presented in Figure 5. It can be noticed that the noise frequency is higher than $15 \mathrm{~Hz}$, which is the reason for choosing the cutoff frequency of $5 \mathrm{~Hz}$.

In the upper part of Figure 6 a raw signal acquired from a gyroscope is presented, while in the lower part the result obtained after applying the digital filtering appears.

\section{Time-domain linear identification}

The structure of an identified model should be as simple as possible in order to represent as accurately as possible the real system. A very complex model can increase the computational load and does not necessarily bring the desired 


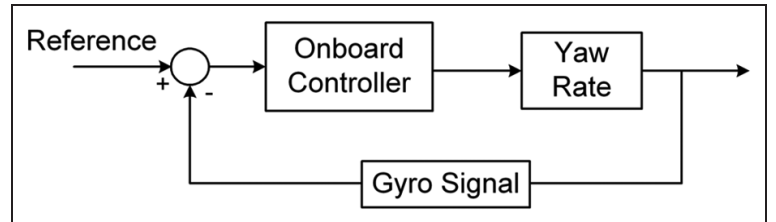

Figure 7. Closed loop yaw rate.

accuracy. Therefore, when choosing a model a compromise has to be made between accuracy and complexity.

Only a few examples of system identification techniques applied to small-scale helicopters exist in the literature, and the results are not as good as in the case of fullscale helicopters. ${ }^{16,21-23}$ Under the assumption that, in hovering, a multivariable helicopter system can be considered as four independent SISO systems, time-domain system identification theory can be used.

In this section linear models are obtained for the helicopter angular movements (yaw, pitch, and roll) from experimental flight data. The method chosen is a linear PEM, also applied in studies by Park et al., Gerig, and Schafroth et al. ${ }^{14,17,24}$ It is a widely used method for system identification, the main idea being to compare the measurement output vector with the predicted output vector. $^{25,26}$

Our goal here is to find a linear time-invariant model and for that reason we construct the parametrized model in the form of $y(t)=G_{\theta}(q) u(t)+H_{\theta}(q) e(t)$, where $y(t), u(t)$, and $e(t)$ are the real output, input, and noise signals, respectively, and $G_{\theta}$ and $H_{\theta}$ are models of the system and noise transfer functions. These transfer functions are parametrized by a real vector $\theta$.

The input-output data in the time domain, used with this method, are obtained after some real test flights. The one-step ahead predictor can be defined as: $\widehat{y}(t \mid \theta)=H_{\theta}(q)^{-1} G_{\theta}(q) u(t)+\left(I-H_{\theta}(q)^{-1}\right) y(t)$, while the prediction error can be defined as $\varepsilon(t, \theta)=y(t)-$ $\widehat{y}(t \mid t-1, \theta)=H_{\theta}(q)^{-1}\left(y(t)-G_{\theta}(q) u(t)\right)$.

The PEM finds the model parameter $\theta$ by minimizing the sample variance of the prediction errors:

$$
\begin{gathered}
V_{N}^{T D}(\theta)=\sum_{k=1}^{N}|\varepsilon(t, \theta)|^{2}=\frac{1}{N} \sum_{k=1}^{N}\left|H_{\theta}(q)^{-1}\left(y(t)-G_{\theta}(q) u(t)\right)\right|^{2} \\
\widehat{\theta}_{N}=\arg \min _{\theta} V_{N}^{T D}(\theta) \\
\widehat{\lambda}_{N}=V_{N}^{T D}\left(\theta_{N}\right)
\end{gathered}
$$

where $\widehat{\lambda}_{N}$ is the estimate of the variance of $e(t)$. The prediction errors are the deterministic system errors $y(t)-G_{\theta}(q) u(t)$ filtered through the inverse of the noise model.
The angular rotation around $z$-axis (yaw) is a particular case for the Big Lama coaxial helicopter. Because of the hardware configuration (see Figure 1), an open loop identification experiment is not possible, since it's almost impossible to keep the helicopter stable for more than a few seconds. Actually, most of the flight tests realized until now by other researchers, were made using a controller to help stabilize the helicopter (especially the yaw rotation) during the experiments, as for example in the study by Schafroth et al. ${ }^{14}$ The internal controller uses the feedback signal of an internal gyroscope to control the yaw rotation (see Figure 7). Figure 7 presents the inner loop of the model described in equation (2).

Therefore, based on the input-output signals presented in Figure 8 and using the PEM method, the following transfer function was obtained for yaw rate:

$$
G_{\text {yaw }}=\frac{241}{s^{2}+614 s+125300}
$$

This transfer function is the result of a closed loop identification, so it incorporates also the onboard controller. It can be noticed that the results are satisfactory. In Figure 8, the output of the process is compared with the output of the linear model, while the validation results of the yaw rate linear model are presented in Figure 9. Even for a different pair of input-output signals the model output behaves very closely to the real system.

\section{Nonlinear modeling}

What recommends the use of neural networks for identification and modeling for nonlinear processes is their capacity to approximate the behaviour of almost any nonlinear process. $^{27}$ This can be achieved by finding the optimal weights and biases, in order to fit the response of the process, by taking into account also the input. The procedure is accomplished by training the neural network based on a training algorithm, in order to minimize the error: $e(k)=y^{*}(k)-y(k)$, where $y^{*}(k)$ is the desired output and $y(k)$ the neural network output.

In this paper nonlinear autoregressive networks with exogenous inputs (NARX) are used to approximate the dynamics of the UAV system. NARX models are based on the linear ARX models, commonly used for time-series modeling. A NARX model is a recurrent dynamic network with feedback connections and can be described by the general equation:

$$
\begin{aligned}
& y(k)=f\left(y(k-1), y(k-2), \ldots, y\left(k-n_{y}\right), u(k-d-1),\right. \\
& \left.u(k-d-2), \ldots, u\left(k-d-n_{u}\right)\right)
\end{aligned}
$$

where $f$ is a nonlinear function, $d$ is the estimated time delay, $n_{y}$ and $n_{u}$ are the orders of the system. Figure 10 presents a simple schematic of a NARX model. 


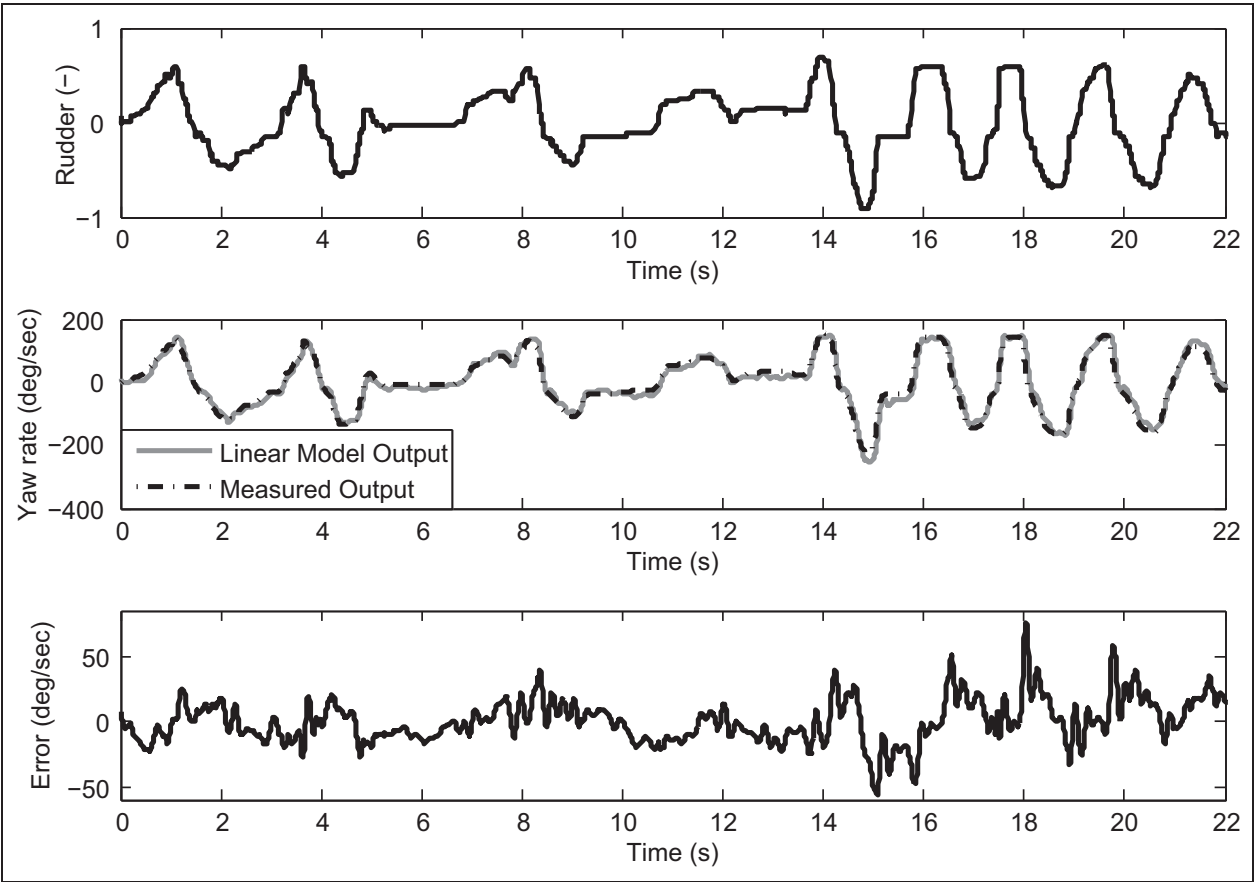

Figure 8. Time-domain yaw rate identification result.

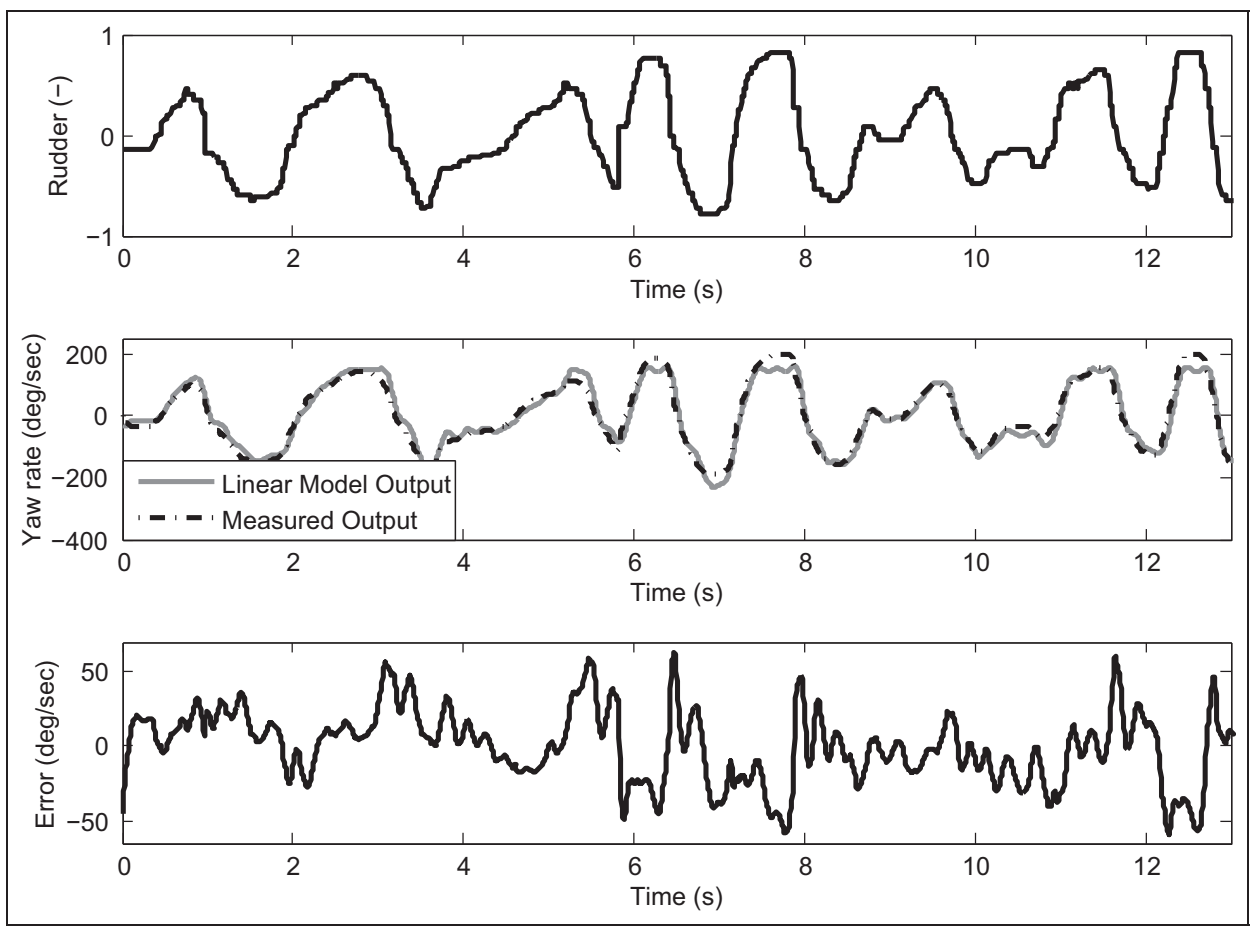

Figure 9. Time-domain yaw rate model validation.

In order to model a nonlinear dynamic system two possible configurations can be used, ${ }^{28}$ the parallel architecture and the series-parallel architecture.
Normally a neural network with a single hidden layer is sufficient to model almost all nonlinear processes. For such a network the output is given by the relation: 


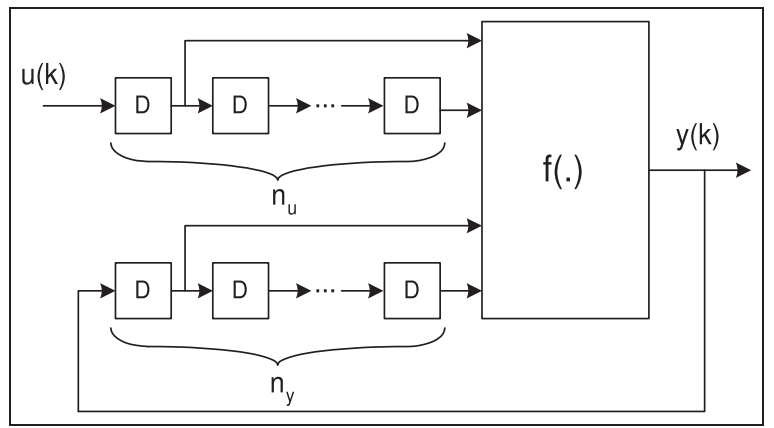

Figure 10. The NARX neural model.

$y(k+1)=\sum_{j=1}^{n_{h}} w_{j} \sigma_{j}\left(\mathbf{w}_{j}^{u} \mathbf{u}(k-d-1)^{\mathrm{T}}+\mathbf{w}_{j}^{y} \mathbf{y}(k-1)^{\mathrm{T}}+b_{j}\right)+b$

$\mathbf{u}(k-d)=\left[u(k-d), u(k-d-1), \ldots, u\left(k-d-n_{u}+1\right)\right]$ $\mathbf{y}(k)=\left[y(k), u(k-1), \ldots, u\left(k-n_{y}+1\right)\right]$

where $n_{h}$ is the number of neurons in the hidden layer, $w_{j}$ is the the weight for the output layer corresponding to the $j$ th neuron from the hidden layer, $\sigma_{j}$ is the activation function of the $j$ th neuron in the hidden layer, $\mathbf{w}_{j}^{u}$ is the weight vector of the $j$ th neuron with respect to the corresponding input vector $u(k-d-1), \mathbf{w}_{j}^{y}$ is the weight vector of the $j$ th neuron with respect to the corresponding output vector $y(k-1), b_{j}$ is the bias of the $j$ th neuron from the hidden layer, and $b$ is the bias of the neuron in the output layer.

The next step of the training process is choosing the parameters $n_{u}, n y$, and $d$ corresponding to the regressed inputs of the neural network. In this phase, accurate information about the process is very important. This information can be obtained from a previous linear identification around an operating point. Furthermore the structure (number of neurons in the hidden layer, number of delays) of the network will be established. The model obtained has to be validated using different input-ouput signals from the ones used for training. If there will be big differences between the process real output and the model output, the steps mentioned above must be repeated (until the desired accuracy is obtained) by choosing different training signals, model structures, training algorithms, and so on.

The steps described previously in this section were applied repeatedly in order to obtain a NARX model for the yaw rate. In Figures 11 and 12 the results obtained after simulating the model are presented. The neural network has 20 neurons in the hidden layer and 4 delays. In the training stage, the Levenberg-Marquard training algorithm was used. Figure 11 presents the training signals and the comparison between the model output and the real ouput, while the results of model validation in Figure 12 appear. It's obvious that the model is very accurate, being able to respond exactly as the physical system. Comparing

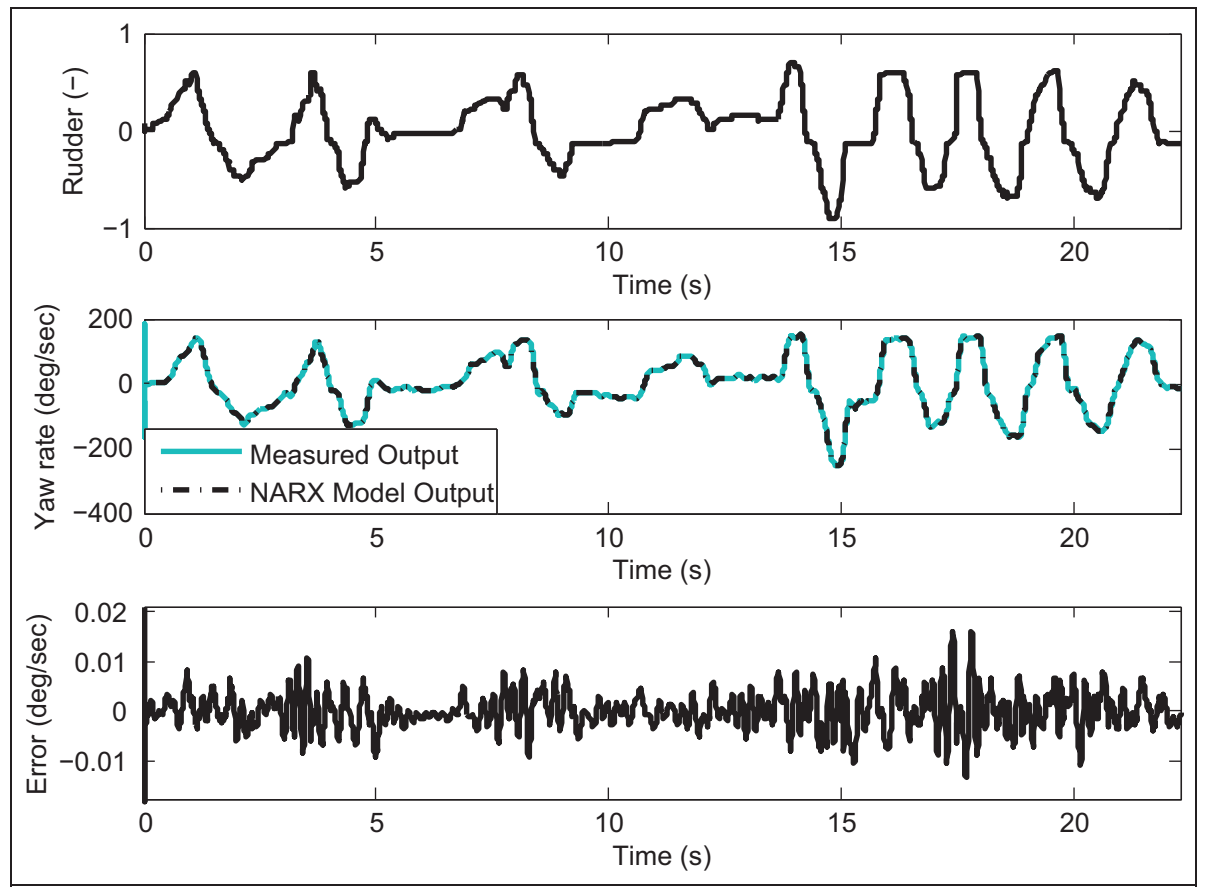

Figure I I. Nonlinear modeling result for yaw rate. 


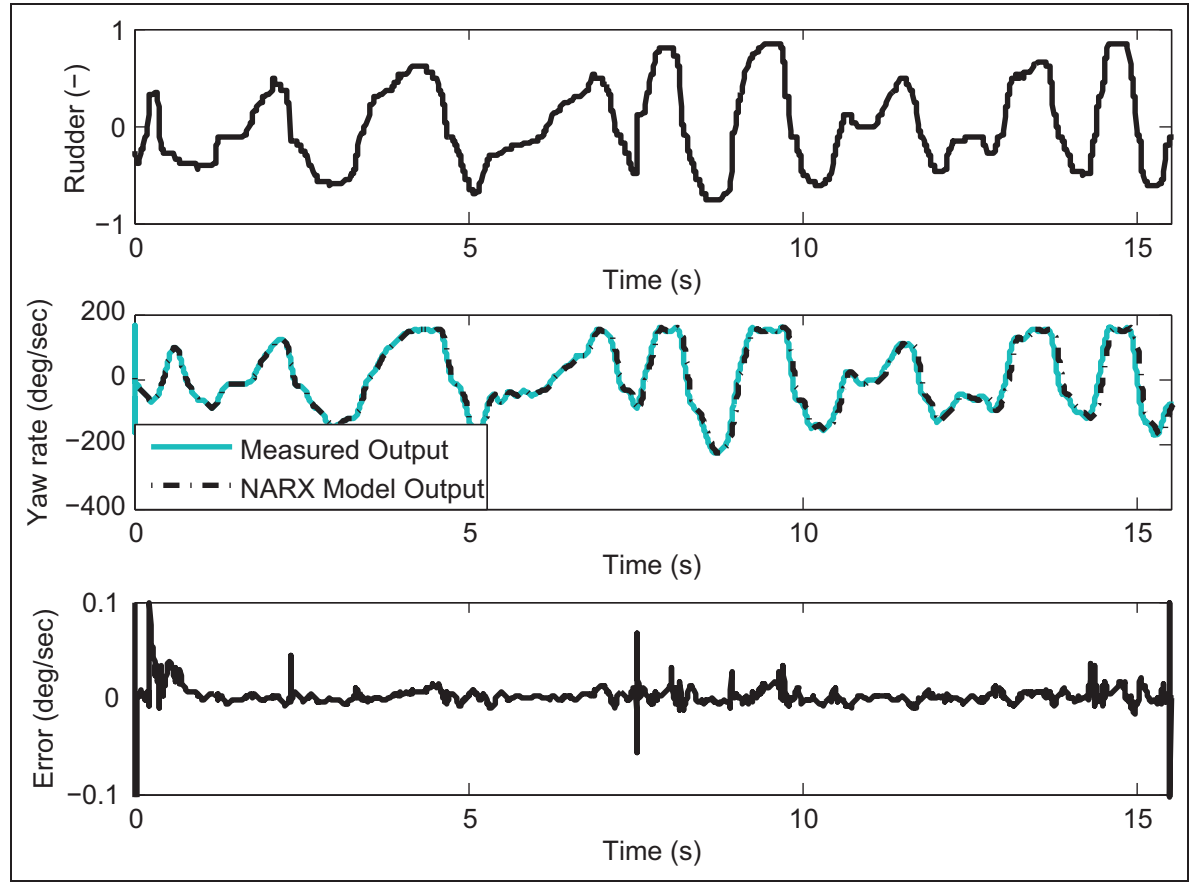

Figure I2. Validation of the nonlinear yaw model.

the linear model error with the nonlinear model error it can be noticed that the difference in magnitude is hundreds of times bigger in the first case.

\section{Results}

In this section the results for the identified models, both linear and nonlinear, for pitch and roll are presented. The time-domain identification for pitch and roll are shown in Figures 13 and 14, and 15 and 16, respectively. Using the PEM method in order to identify the roll rate, the transfer function below was obtained:

$$
G_{\text {roll }}=\frac{-1277.8 s-379.87}{s^{2}+5.87 s+76.9}
$$

In Figure 13 the true output is compared with the model output, resulted for the same input signal, while in Figure 14 the two responses are compared for a different input signal in order to validate the linear model.

Using the same PEM method for pitch, the following transfer function resulted:

$$
G_{\text {pitch }}=\frac{1108 s+664.9}{s^{2}+5.43 s+66.2}
$$

Figures 15 and 16 present the responses of both the model and the real process, in the case of pitch rate, for two different input signals.
In general the models outputs present a good estimation of the responses of the real system. There are only small deviations in amplitude especially, but this is not critically for control design because it can be fixed by the controller's gain. The accuracy of the models is comparable with the ones obtained by Schafroth et al. ${ }^{14}$ It has to be noticed that it's a great challenge to perform flight tests for longer than $20 \mathrm{~s}$ because the helicopter is unstable and difficult to maneuver in very long open-loop tests. But despite this drawback the models obtained are accurate enough to make possible the design of a proper controller.

Figures 17 and 18, and 19 and 20, respectively, show the results of nonlinear modeling using neural networks. From the beginning it can be seen that the accuracy of the NARX models is much higher than the ones in the linear case. Actually, hardly any difference can be seen between the model's outputs and the real system's outputs. Even in the validation process, for different pairs of input-output signals, the estimation is very good.

A NARX network was trained using again the Levenberg-Marquardt algorithm in order to approximate the behaviour of the roll rate. The structure of the neural network based models (number of neurons per layer, number of delays) was chosen by experiment because there is no proved technique that allows for an accurate calculation. Therefore, the chosen neural model has 1 hidden layer with 15 neurons and 4 unit delays. The response of 


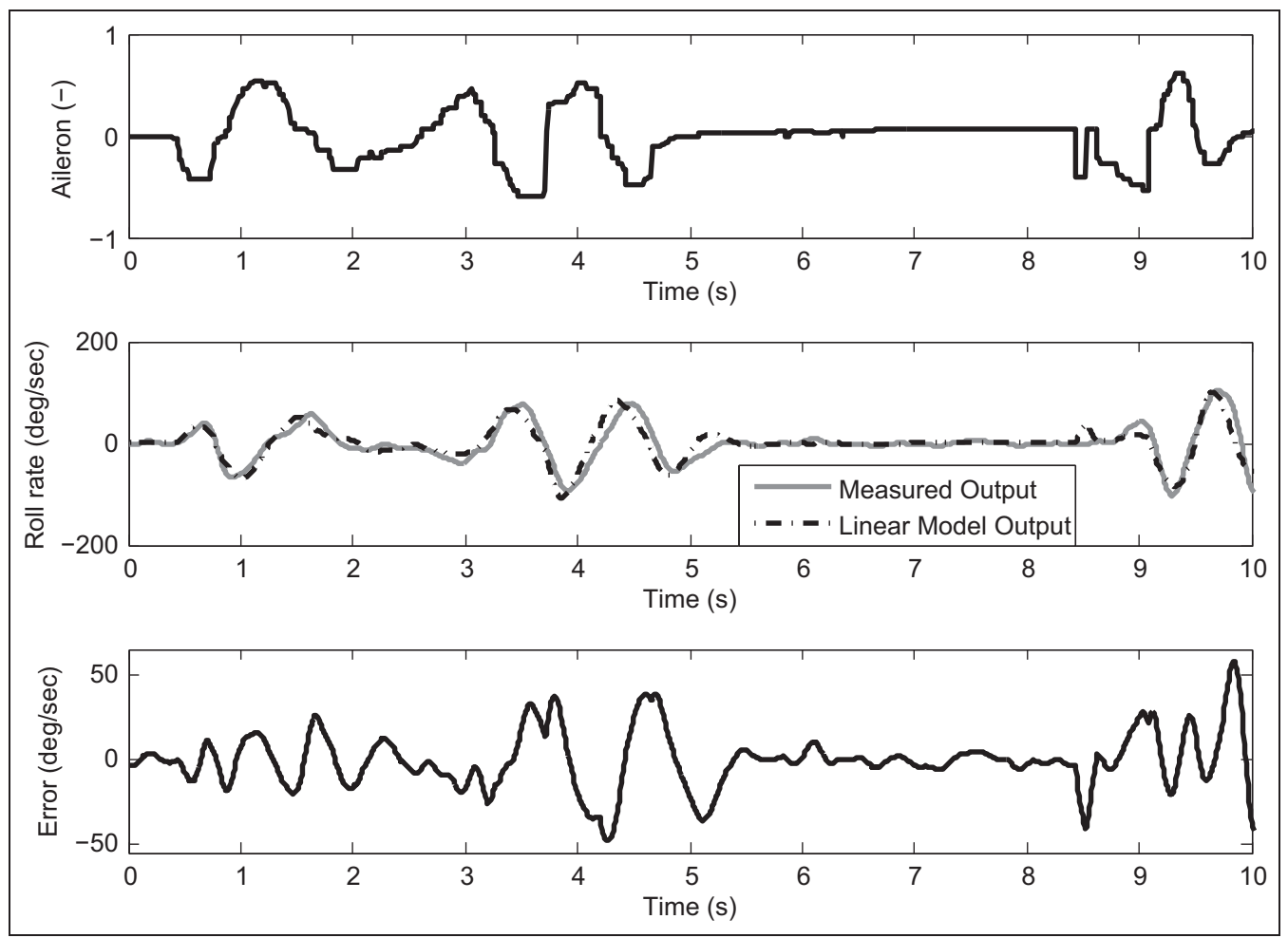

Figure 13. Time-domain roll rate identification result.

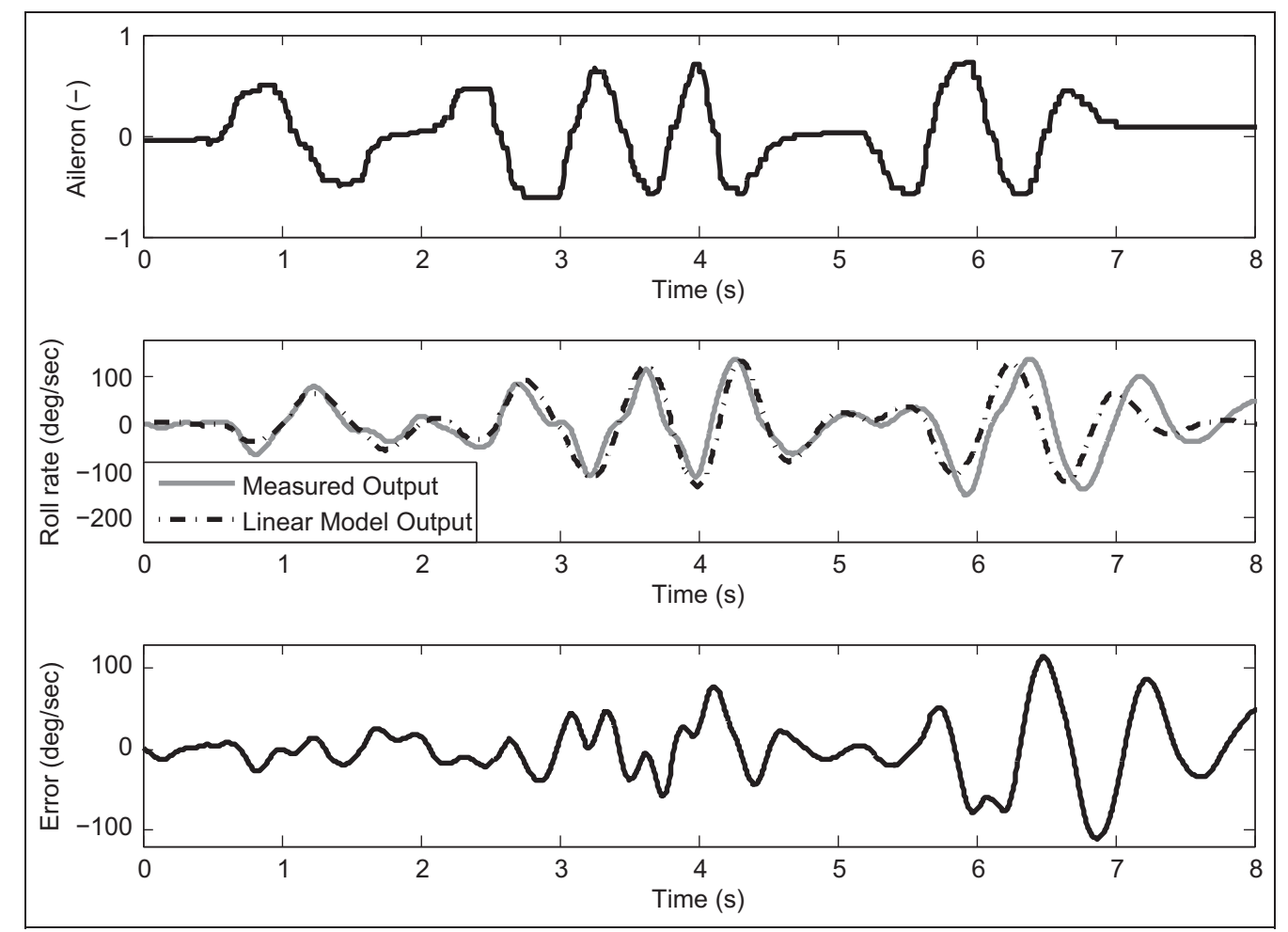

Figure 14. Time-domain roll rate model validation. 

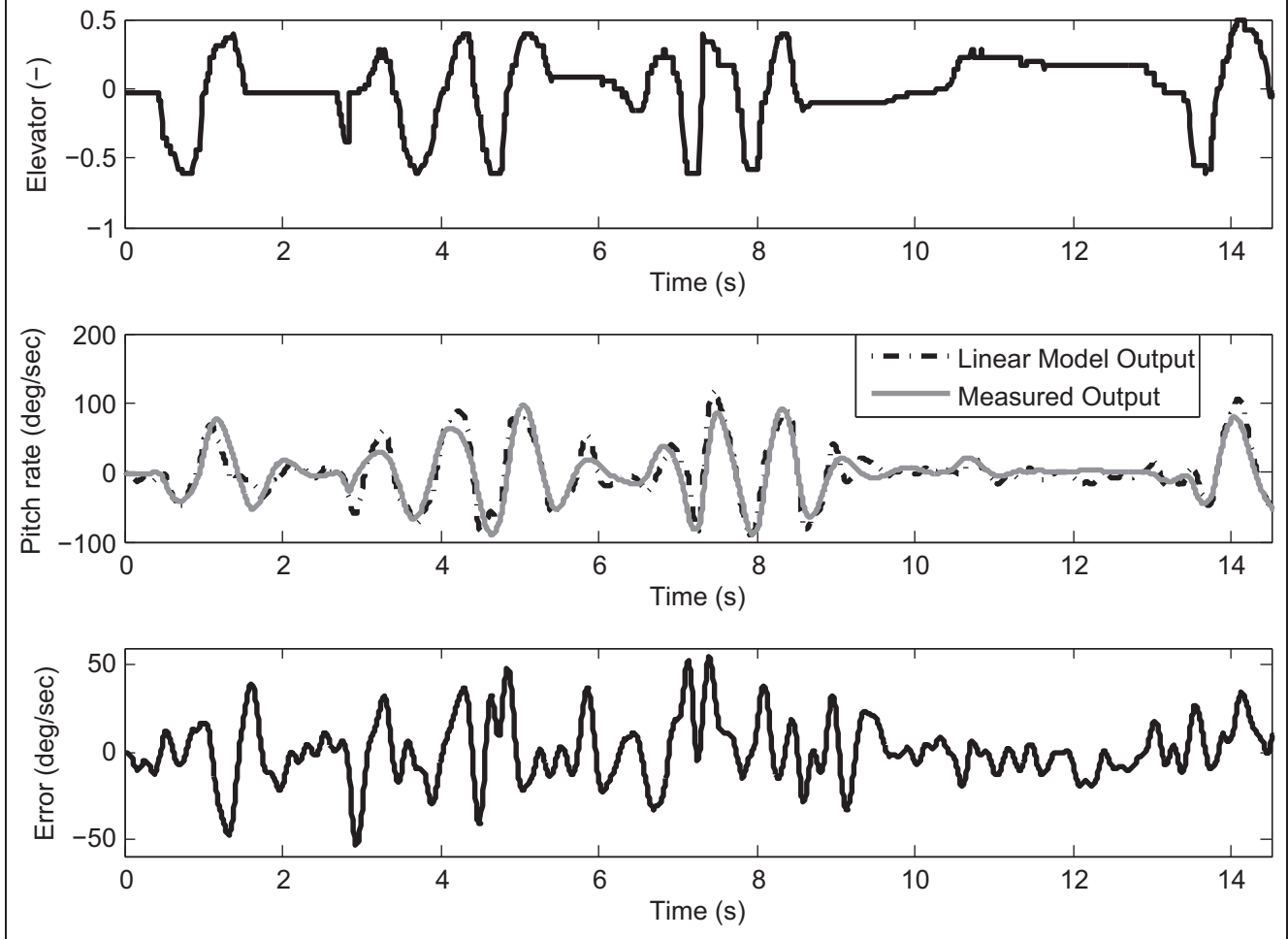

Figure 15. Time-domain pitch rate identification result.

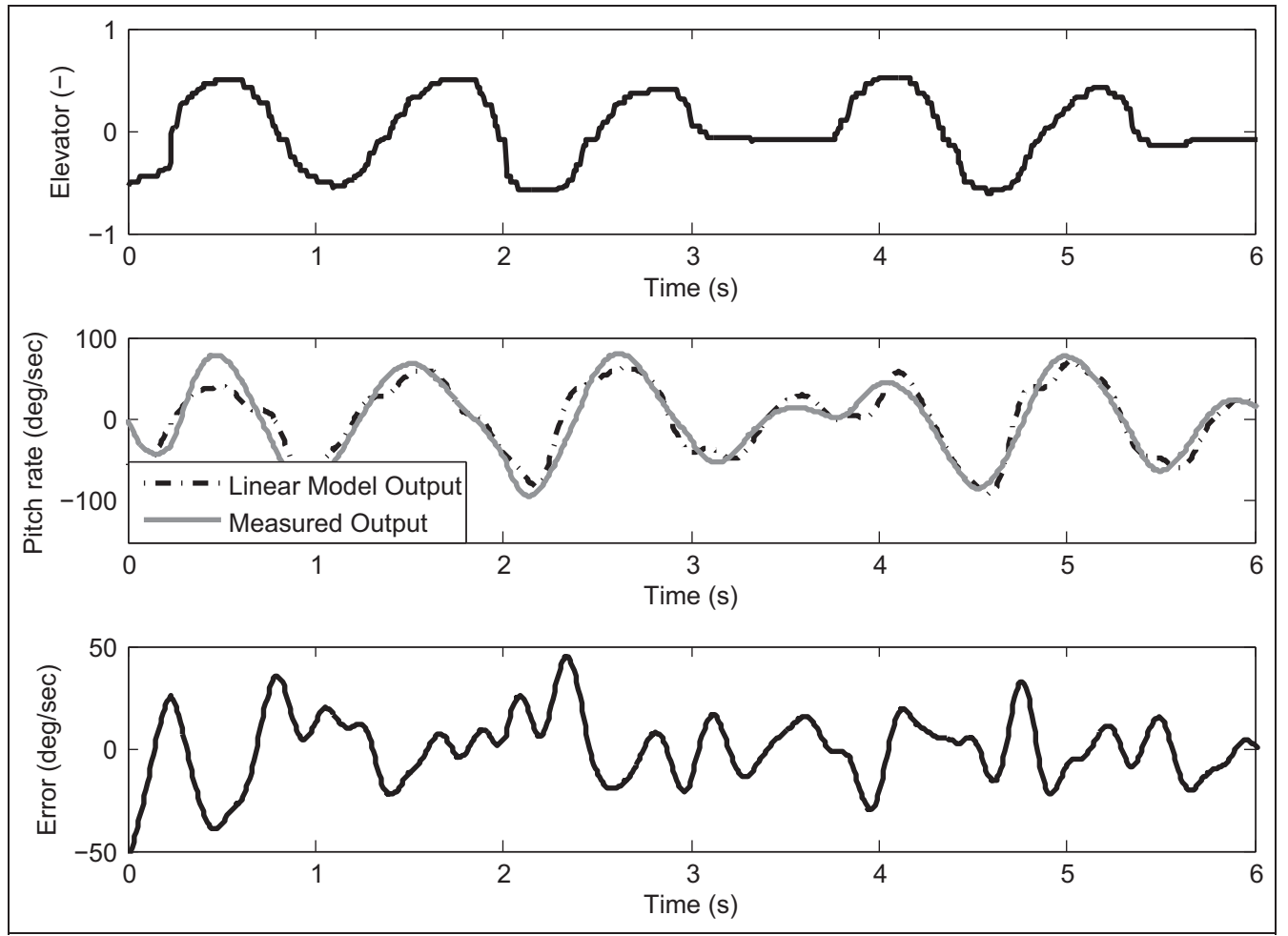

Figure 16. Time-domain pitch model validation. 


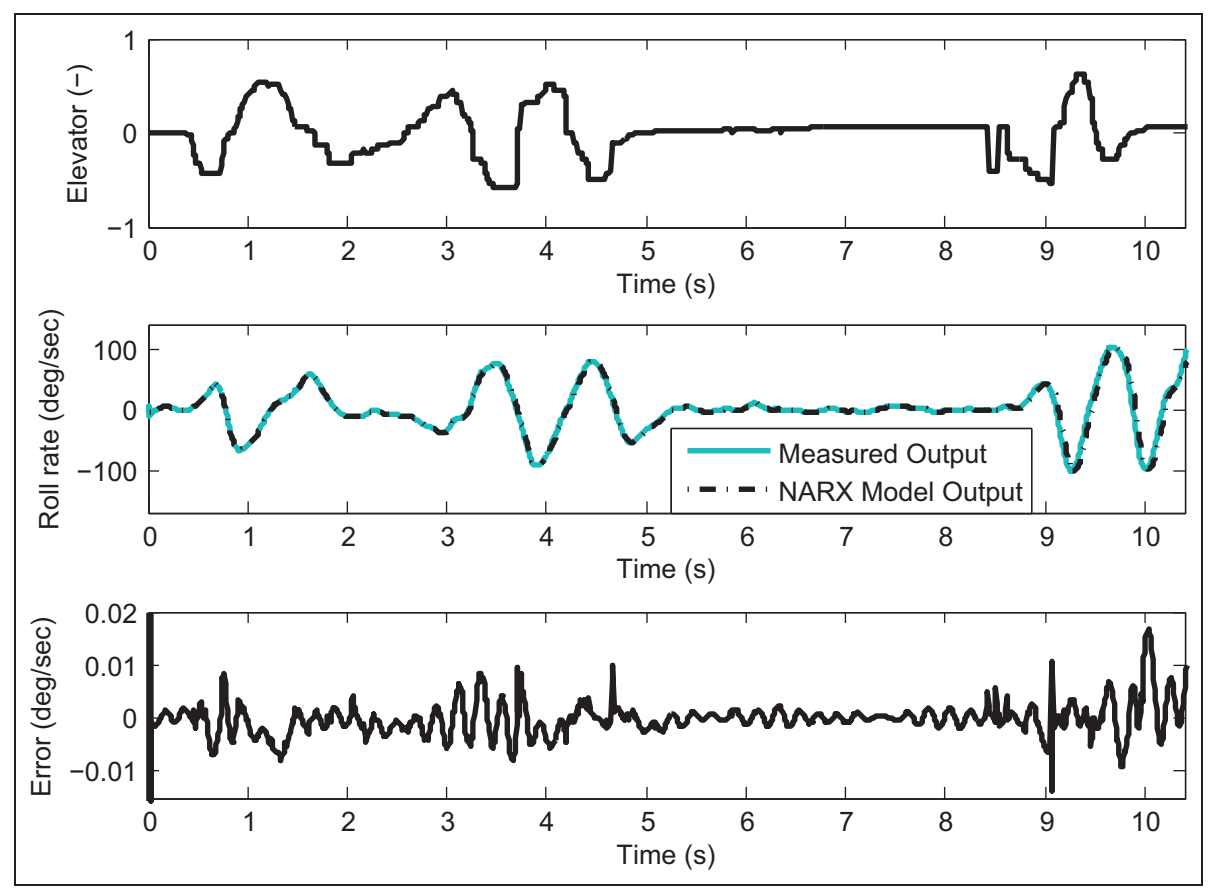

Figure 17. Nonlinear modeling result for roll rate.

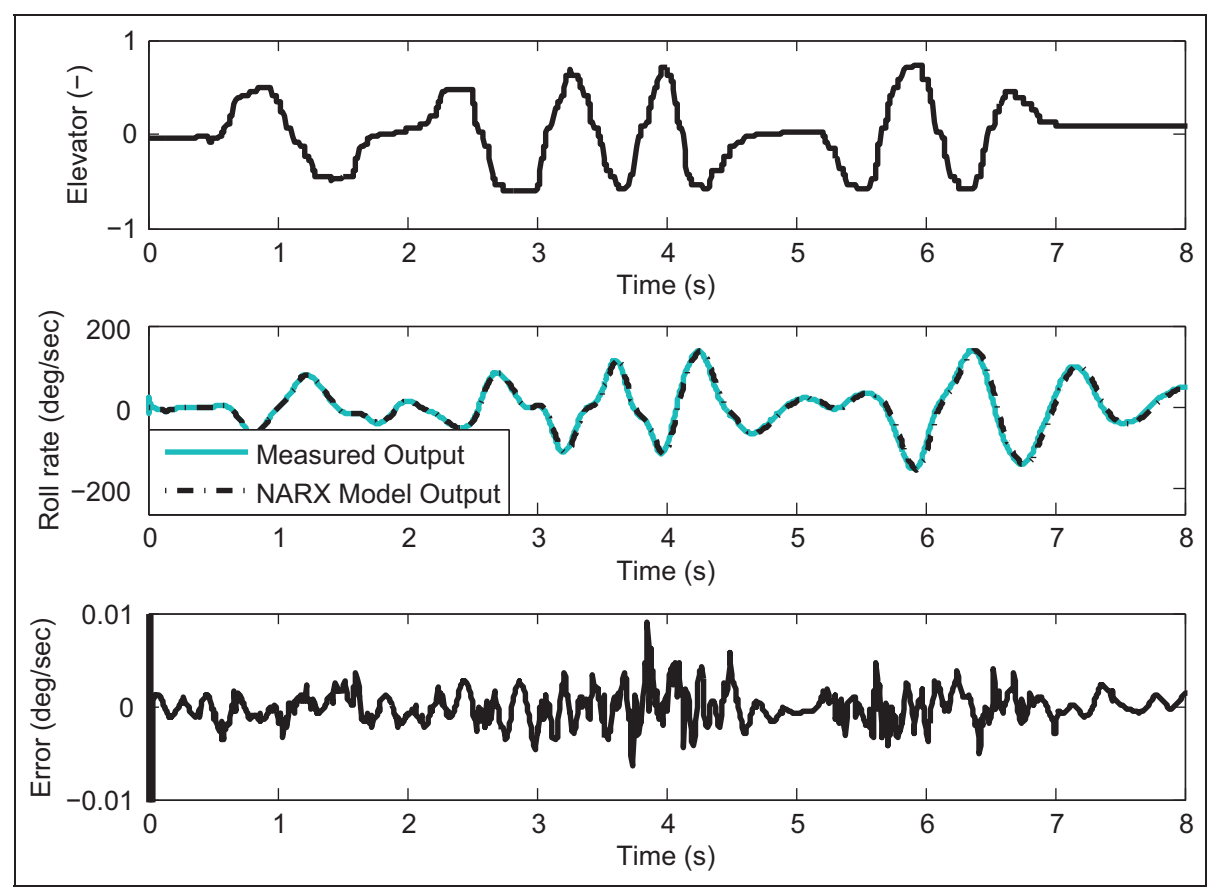

Figure 18. Validation of nonlinear roll rate model.

the model is compared below with the response of the real process to the same input signal.

The neural model chosen for pitch has one hidden layer with twelve neurons and four unit delays. The results are presented in Figures 19 and 20.

\section{Discussion and conclusions}

In this paper both linear and nonlinear models are developed for the three rotation movements of a miniature coaxial helicopter. 


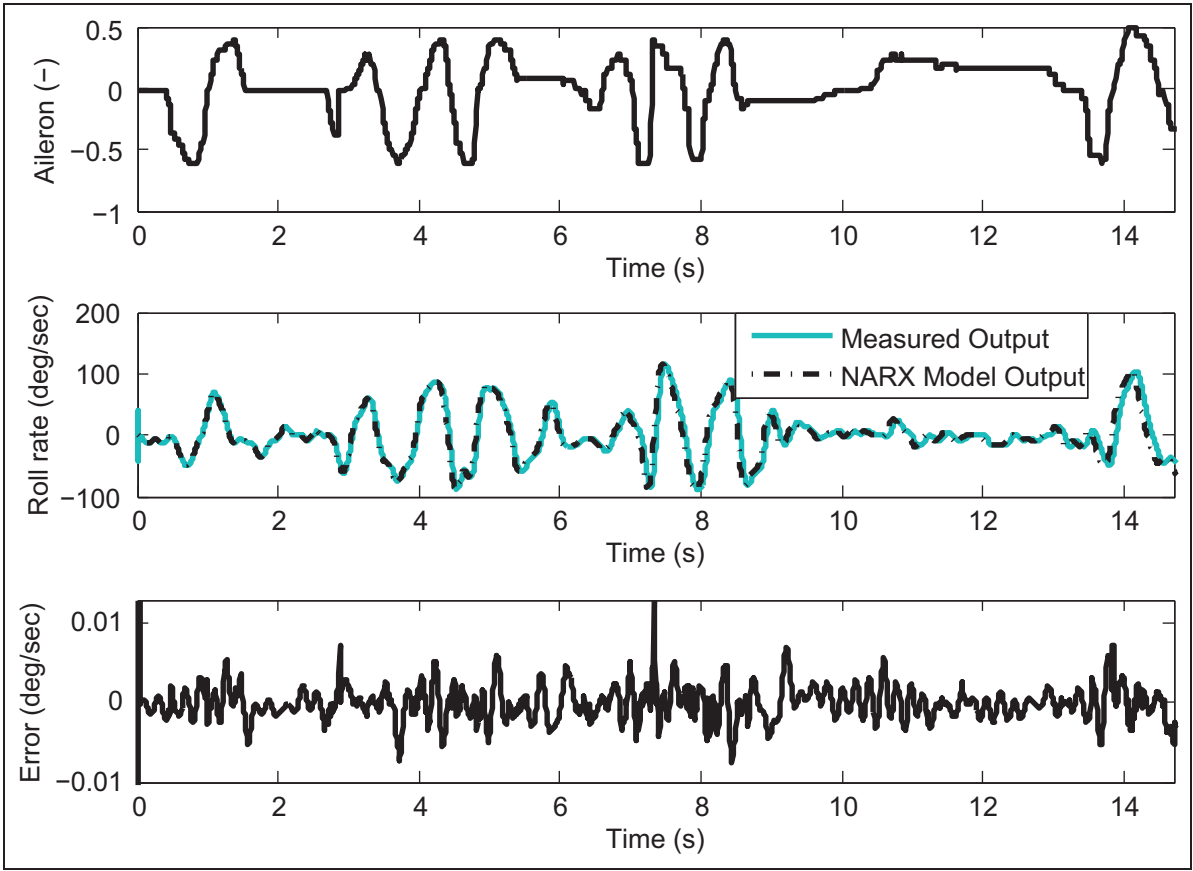

Figure 19. Nonlinear modeling result for pitch rate.

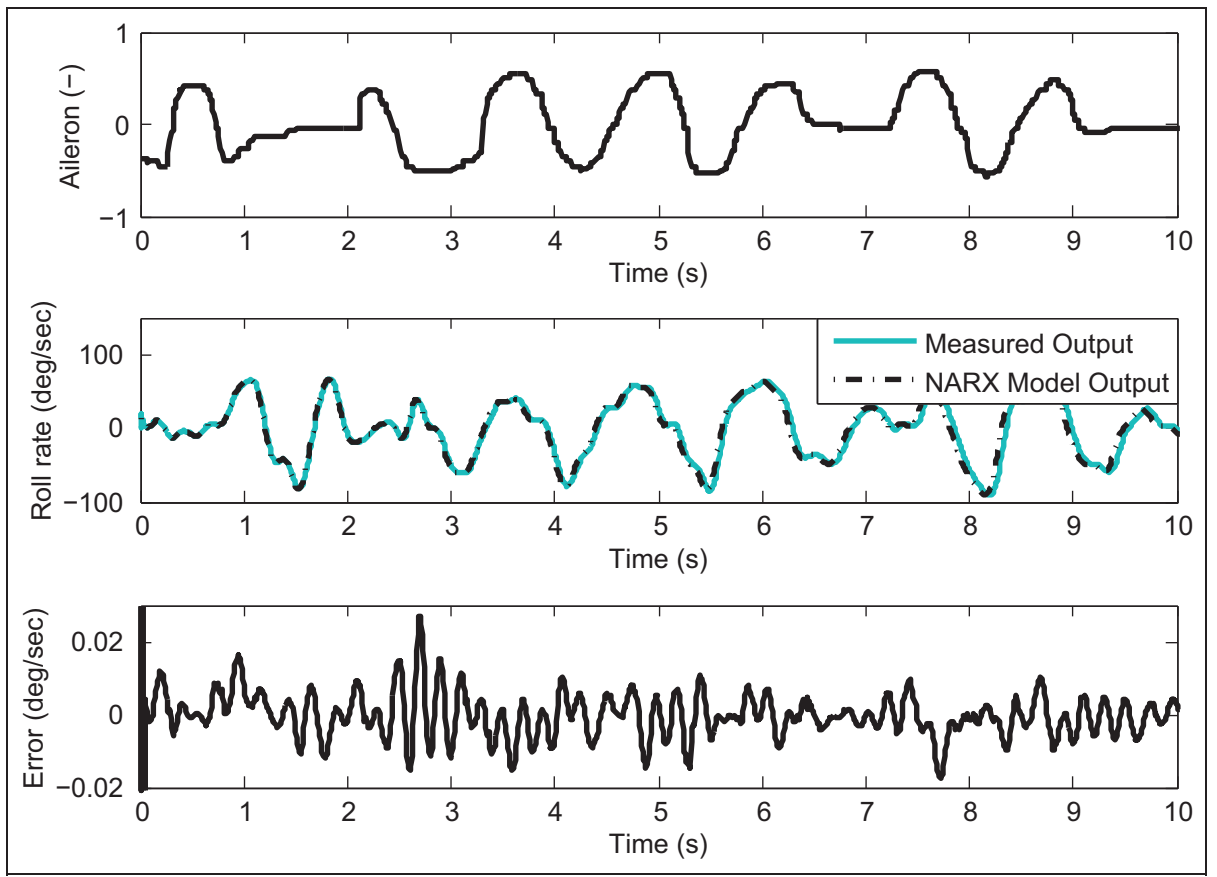

Figure 20. Validation of nonlinear pitch rate model.

A low cost UAV platform was developed using an already available coaxial helicopter and a Tag4M module. Besides the financial issue, the configuration obtained presents several other advantages like the wireless communication and analog and digital input-output available which makes the platform flexible, i.e. sensors can be added on the board, data can be acquired in real-time during flight tests and controllers can be implemented using 
the digital outputs to send the PWM signals to the actuators. Using software applications developed in LabVIEW and Matlab, the study of the helicopter dynamics was possible.

Based on the flight data, linear models were obtained using a PEM and the accuracy is high enough to design performant controllers. The results are better than the ones presented in the study by Schafroth et al. ${ }^{14}$ Even so, they include only the dynamics of the helicopter around an operation point (in this case hovering).

Further on, nonlinear models were developed based on neural networks. The neural networks-based modeling can be applied successfuly as an alternative to the linear modeling or the first principle modeling because their ability to approximate the dynamics of a nonlinear system on all the operating range. For this reason NARX networks were trained using the real sensor data. Their performances are superior compared not only to the linear ones, but also compared to the performances obtained in studies by Suresh et al. and San Martin et al. ${ }^{20,29}$ The drawback in the case of this kind of model is that no model-based control algorithms can be implemented. But, since one of the purposes of the research project is to design a predictive controller based on neural models, the results obtained at this point are promising.

In the next stage of the project, the authors' plan is to design, develop, and validate flight embedded controllers based first on the linear models and then on nonlinear models (neural models).

\section{Funding}

This work was supported by POSDRU/6/1.5/S/5 (grant number 7676).

\section{References}

1. Budiyono A. Recent advances in control and instrumentation of unmanned aerial vehicles. In: Conference on instrumentation and control, Bandung, Indonesia, 19-20 February 2007.

2. Meyer J, de Plessis F and Clarke W. Design considerations for long endurance unmanned aerial vehicles. In: Thanh Mung Lam (ed) Aerial Vehicles. New York: Intech Publishing, 2009, pp.443-497.

3. Kumar MV, Suresh S, Omkar SN, Ganguli R and Sampath P. A direct adaptive neural command controller design for an unstable helicopter. Engineering Applications of Artificial Intelligence 2009; 22: 181-191.

4. Wang H, Mian AA, Wang D and Duan H. Robust multi-mode flight control design for an unmanned helicopter based on multi-loop structure. International Journal of Control, Automation, and Systems 2009; 7(5): 723-730.

5. Verhoeven GJJ, Loenders J, Vermeulen F and Docter R. Helikite aerial photography - A versatile means of unmanned, radio controlled, low-altitude aerial archaeology. Archaeological Prospection 2009; 16(2): 125-138.
6. Torres $\mathrm{G}$ and Mueller T. Micro air vehicle development: Design, components, fabrication, and flight-testing. In: AUVSI unmanned systems 2000 symposium and exhibition, Orlando, FL, 11-13 July 2000.

7. Wu HY, Sun D and Zhou ZY. Micro air vehicle: Configuration, analysis, fabrication and test. IEEE/ ASMETrans Mechatronics 2004; 9(1): 108-117.

8. Lei $\mathrm{X}$ and Du Y. A linear domain system identification for small unmanned aerial rotorcraft based on adaptive genetic algorithm. Journal of Bionic Engineering 2010; 7: 142-149.

9. Bouabdallah S, Becker M and Siegwart R. Autonomous minituare flying robots: Coming soon! IEEE Robotics and Automation Magazine 2007; 14(3): 88-98.

10. Schafroth D, Bouabdallah S, Bermes C and Siegwart R. From the test benches to the first prototype of the muFly micro helicopter. Journal of Intelligent and Robotic Systems 2008; 54: 245-260.

11. Escareño J, Sanchez A, Garcia O and Lozano R. Modeling and global control of the longitudinal dynamics of a coaxial convertible mini-UAV in hover mode. Journal of Intelligent and Robotic Systems 2009; 54: 261-273.

12. Calise AJ and Rysdyk RT. Nonlinear adaptive flight control using neural networks. IEEE Controls Systems Magazine 1998; 18(6): 14-25.

13. Savran A, Tasaltin R and Becerikli Y. Intelligent adaptive nonlinear flight control for a high performance aircraft with neural networks. ISA Transactions 2006; 45(2): 225-247.

14. Schafroth D, Bouabdallah S, Bermes C and Siegwart R. Modeling and system identification of the muFly helicopter. Journal of Intelligent and Robotic Systems 2009; 57: 27-47.

15. Schafroth D, Bermes C, Bouabdallah S and Siegwart R. Modeling, system identification and robust control of a coaxial microhelicopter. Control Engineering Practice 2010; 18: $700-711$.

16. Mettler B, Tischler MB and Kanade T. System identification of small-size unmanned helicopter dynamics. In: American helicopter society 55th forum, Montreal, Quebec, 25-27 May 1999.

17. Park D, Park MS and Hong SK. A study on the 3-DOF attitude control of free-flying vehicle. In: IEEE international symposium on industrial electronics, Pusan, South Korea, 12-16 June 2001, Vol. 2, pp.1260-1265.

18. Folea S and Ghercioiu M. Tag4M, a Wi-Fi RFIDActive tag optimized for sensor measurements. In: Cristina Turcu (ed) Radio Frequency Identification Fundamentals and Applications Design Methods and Solutions. New York: InTech Education and Publishing, 2010, pp.287-310.

19. Kumar R, Ganguli R and Omkar SN. Rotorcraft parameter estimation using radial basis function neural network. Applied Mathematics and Computation 2010; 216(2): 584 597.

20. San Martin R, Barrientos A, Gutierrez P and del Cerro J. Unmanned aerial vehicle (UAV) modelling based on supervised neural networks. In: IEEE international conference on robotics and automation, Orlando, FL, 15-19 May 2006, pp.2497-2502.

21. Mettler B, Kanade T and Tischler MB. System identification modeling of a model-scale helicopter. Working paper 
CMU-RI-TR-00-03, Robotics Institute, Carnegie Mellon University, Pittsburgh, PA, 2000.

22. Adiprawita W, Ahmad AS and Sembiring J. Automated flight test and system identification for rotary wing small aerial platform using frequency responses analysis. Journal of Bionic Engineering 2007; 4: 237-244.

23. Velagi $\mathrm{J}$ and Osmin N. Identification and control of 2DOF nonlinear helicopter model using intelligent methods. In: IEEE International conference on systems man and cybernetics (SMC), Istanbul, Turkey, 10-13 October 2010, pp.2267-2275.

24. Gerig M. Modeling, guidance, and control of aerobatics maneuvers of an autonomous helicopter. $\mathrm{PhD}$ Diss, Eidgenössische Technische Hochschule ETH Zürich, 2008.

25. Ljung L. System identification: Theory for the user. Upper Saddle River, NJ: Prentice-Hall, 1987.

26. McKelvey T. Relations between time domain and frequency domain prediction error methods. In: Encyclopedia of Life Support Systems (EOLSS) (Vol. V, Control systems, robotics, and automation), 2004.

27. Hornik K, Stincombe M and White H. Universal approximation of an unknown mapping and its derivatives using multilayer feedforward networks. Neural Networks 1990; 3: 211223.

28. Narendra KS and Parthasarathy K. Identification and control of dynamical systems using neural networks. IEEE Transactions on Neural Networks 1990; 1(1): 4-27.

29. Suresh S, Kumar MV, Omkar SN, Mani V and Sampath P. Neural networks based identification of helicopter dynamics using flight data. In: Proceedings of the 9th international conference on neural information processing, Singapore, 18-22 November 2002, Vol. 1, pp.10-14.

\section{Author biographies}

Bogdan Muresan received a BSc degree in Automation and Applied Informatics in 2008 and a $\mathrm{PhD}$ degree in Systems Engineering in 2011 from the Technical University of Cluj-Napoca, Romania. In 2009, he obtained also a Master's degree in Business Administration from Babes-Bolyai University, Cluj-Napoca, Romania. Between 2008 and 2011, he was a researcher and teaching assistant at the Technical University of Cluj-Napoca, Romania. His main interests include: predictive control, neural networks, system identification and modeling, and miniature coaxial helicopters. Since 2011, he is working in the industry for a top multinational company in the distributed control systems field.

Silviu C Folea received a BSc degree in control systems from Technical University of Cluj-Napoca, Romania, in 1995 and $\mathrm{ahD}$ in 2005. He is currently associate professor in the Automation Department at the Technical University of Cluj-Napoca, Romania. His research interests include: embedded systems-hardware and software, reconfigurable systems, data acquisition, wireless networks, and low power sensors; his software interests include LabVIEWTM graphical programming for Real Time or FPGA modules with courses taught at Bachelor's and Master's degree level. Professor Folea has 17 years of design experience in the embedded systems domain and in university teaching. He is the author of nine books and book chapters and around 80 conference and journal publications, and has edited contributed volumes. Professor Folea has taken part in more than 30 research contracts: international, national, and with external industry partners.

Ioan Nascu received a License degree in 1983 from Polytechnic Institute of Cluj-Napoca and a $\mathrm{PhD}$ degree in 1997 from the Technical University of Cluj-Napoca, Romania, both in Control Systems. Presently he is professor in the Automation Department of the Technical University of Cluj-Napoca. His research interests include topics such as modeling and control of industrial processes, predictive control, and adaptive control.

Clara $\mathbf{M}$ lonescu received an MSc degree in Industrial Informatics and Automation from Dunarea de Jos University, Galati, Romania, in 2003. She obtained a PhD degree at Ghent University, Ghent, Belgium, in 2009, studying the identification of the human respiratory system by means of fractional order models. Currently, she is a post-doc fellow in the same university, involved in several international projects, with both industrial and biomedical applications, for identification and control. Her main research interests include biomedical applications, with identification and advanced control objectives. Dr. Ionescu is the holder of the prestigious post-doctoral grant from Flanders Research Foundation (FWO).

Robin De Keyser obtained an MSc degree in ElectroMechanical Engineering in 1974 and a $\mathrm{PhD}$ degree in Control Engineering in 1980 from Ghent University, Belgium. He is currently full Professor of Control Engineering at the Faculty of Engineering, Ghent University. He is author/co-author of arount 300 publications, including journals, books, and conference proceedings. He acted as external review expert in several European Commission research programs and is one of the pioneers who produced the original concepts of predictive control during the 1980s. His teaching and research activities include model predictive control, auto-tuning and adaptive control, modeling and simulation, and system identification. His research is application-driven, with many pilot implementations in technical and non-technical systems, among others chemical, steel, marine, mechatronic, semiconductor, and power electronics and biomedical. 